\title{
Protée
}

\section{La lecture littéraire comme parcours dans l'aire du dire : prolégomènes à une sémiotique de la réception}

\section{Nicolas Xanthos}

Volume 27, numéro 2, 1999

La réception

URI : https://id.erudit.org/iderudit/030557ar

DOI : https://doi.org/10.7202/030557ar

Aller au sommaire du numéro

Éditeur(s)

Département des arts et lettres - Université du Québec à Chicoutimi

ISSN

0300-3523 (imprimé)

1708-2307 (numérique)

Découvrir la revue

Citer cet article

Xanthos, N. (1999). La lecture littéraire comme parcours dans l'aire du dire : prolégomènes à une sémiotique de la réception. Protée, 27(2), 35-44.

https://doi.org/10.7202/030557ar
Résumé de l'article

Questionnant le caractère singulier qu'on suppose à la lecture littéraire, le présent article vise à explorer les points de convergence entre la réflexion sur la réception et la réflexion sur la sémiotique. Au prix d'une mise en rapport patiente des concepts, semble-t-il voisins, de paradigme chez Kuhn et de jeu de langage chez Wittgenstein, on en vient à poser les pratiques sémiotiques comme véhiculant des manières de voir implicites qui ouvrent, en le délimitant, un espace de discours et d'action. La singularité des hypothèses de lecture est ainsi repensée au sein de cette conception particulière, et une certaine sémiotique semble, du coup, à même de fournir un cadre heuristique pour l'étude de la réception. 


\section{LETCTUS LA LECTURELTTÉRARE COMMEPARCO URSDANSL'AIREDU DIRE: PRO LÉGO M ÈNES À UNE SÉMIOTIQ UE DE LA RÉCEPTION ${ }^{1}$}

NICOLAS XANTHOS

\section{INTRODUCTION}

S'il est un champ de recherche dont les modalités et les objets d'investigation sont multiples, c'est bien celui des théories de la réception. Dans le sillage de ces figures de proue que sont, par exemple, Hans Robert Jauss et ses défis historiques à la théorie littéraire ou encore Wolfgang Iser et sa Leerstelle, on trouve plusieurs autres voies navigables, inégalement fréquentées. Dans une communication déjà ancienne, Rita Schober (1983) dénombrait sept questions différentes englobées par la problématique de la réception: la fortune des œuvres, auteurs et courants littéraires; «l'histoire littéraire en tant que "histoire propre” grâce à l'activité réceptive des lecteurs comme facteurs constitutifs du processus historique»; «la différentiation (sic) entre divers types de réception»; «la stratégie de réception et d'effet»; «l'appropriation active et, par conséquent, transformatrice de l'œuvre de la part du lecteur"; «l'interaction dialectique entre l'œuvre en tant que Rezeptionsvorgabe (modèle ou projet de réception) et le lecteur en tant que récepteur-transformateur"; «les conséquences de la réception, voire la "fonction sociale" que l'œuvre littéraire assume» (p.7). Il faudrait sans doute évaluer avec précision les fondements et l'exhaustivité de ces diverses catégories; néanmoins, elles indiquent bien le caractère pluridimensionnel du domaine de recherche que recouvre la dénomination fédératrice de réception.

C'est dire qu'une réflexion sur la réception, sauf ambition panoramique, doit commencer par pointer du doigt la portion de ce vaste territoire dont elle entend prendre la mesure. Le présent article, qui examine les limites de la singularité dans la lecture littéraire, cherche plus fondamentalement à poser les jalons d'un questionnement sémiotique de la réception où l'acte de lecture est pensé comme fonction d'une architecture conceptuelle implicite qui l'organise silencieusement ${ }^{2}$. À la suite de Wittgenstein (1961 et 1965), nous estimons que nos manières de nous servir du langage sont tributaires de soubassements implicites d'ordre conceptuel, appris dans et par l'action et soustraits au regard de qui est familier avec les manières en question. La leçon est d'importance et nous semble fournir un cadre fécond pour l'analyse de la réception en général, et de la lecture littéraire en particulier. Dans ce cadre, cette dernière peut être envisagée 
comme une pratique qui prend appui sur une manière spécifique de penser l'objet texte et l'élaboration de la signification. Car produire une lecture littéraire, c'est effectuer sur le texte un certain nombre d'opérations sémiotiques qui ne valent que d'être inscrites dans l'aire ouverte par les concepts implicites de texte et de signification sur lesquels repose la pratique. La réflexion que nous proposons vise à penser la lecture littéraire comme ouverture et délimitation de l'aire du dire par le biais de concepts implicites. Défendant cette idée, nous essayons de mettre en place les prolégomènes d'une sémiotique de la réception.

La question précise dont nous allons débattre peut s'esquisser ainsi: en raison de sa mise à distance d'enjeux théoriques généralisants, la lecture littéraire se présente souvent comme un rapport au texte s'établissant sur le mode de la singularité et de la responsabilité du lecteur. Et c'est ce lien que nous voudrions interroger. Il n'est pas question de nier la distinction évidente entre la lecture littéraire et les autres types d'analyses fondés sur une méthodologie stricte; mais cette distinction n'est garante que d'une certaine forme de singularité des hypothèses de lecture, une singularité que l'on qualifiera de réglée. Celle-ci détermine les conditions de réception du texte. Et, contrairement à ce que l'on pourrait supposer, parler de singularité réglée n'est pas une manière de diminuer l'intérêt de la lecture littéraire comme pratique sémiotique ni de minimiser sa portée. C'est bien plutôt une façon de la situer au plus près de certains enjeux fondamentaux de cet acte complexe qu'est le travail sur le texte.

Un détour patient par Thomas Kuhn et Ludwig Wittgenstein sera nécessaire pour dessiner les contours des prémisses sémiotiques d'un questionnement sur la réception. Nous pourrons par la suite reconsidérer dans ce cadre la singularité des hypothèses de lecture et la responsabilité du lecteur et souligner les enjeux essentiels de la lecture littéraire comme manière de faire sens. Au sortir de ce parcours nous dégagerons les lignes directrices d'une sémiotique de la réception.
POINT DE VUE SUR LA LECTURE LITTÉRAIRE

Avant d'en venir à ces analyses, nous voudrions dire quelques mots de notre objet. Pour ce faire, nous allons synthétiser brièvement les principales idées présentées par Bertrand Gervais dans son ouvrage Lecture littéraire et exploration en littérature américaine, portant sur la pratique qui nous intéresse.

La lecture littéraire diffère à la fois de la lecture courante et de la lecture savante. Contrairement à la lecture courante qui vise la saisie informative minimale du texte, la lecture littéraire est un investissement particulier dans le texte, une «exploration de certains de ses aspects, qu'ils soient langagiers, structuraux, symboliques ou sociaux» (1997 :26). Par suite de cet investissement accru, elle fait l'objet d'une mise en discours: minimalement, elle est intervention sur le texte, qui se signale par les notes dans la marge ou sur la couverture du livre. Et, à l'autre extrême, elle prend la forme d'article, de thèse, d'essai, etc. La lecture littéraire se distingue également de la lecture savante, par le fait qu'elle pose la singularité du lecteur et du texte. C'est du rapport aux théories et méthodologies littéraires qu'il est question ici. En lecture savante, le texte devient souvent un moyen de vérifier des hypothèses théoriques générales; de la sorte, tel texte particulier permet, par exemple, d'observer une instanciation parmi d'autres de la séquence narrative. La théorie ou la méthodologie ainsi expérimentée impose au lecteur «ses choix épistémologiques [...], sa perspective, [...] sa propre conception du texte» (p.35). En lecture littéraire, le texte est pris pour lui-même, imposant «de par sa spécificité les enjeux théoriques ou critiques développés» (p.37), et le lecteur doit assumer la responsabilité du cadre de référence et des modèles qu'il élabore pour rendre compte du texte.

Se joue alors une double remise en question des savoirs du lecteur. D'une part, même si ce dernier peut prendre appui sur les théories et méthodologies littéraires existantes, le texte sur lequel il réfléchit ne vise pas à les confirmer. D'autre part, le point de départ d'une lecture littéraire, de cet investissement particulier dans le texte, est un problème de lecture: 
on s'y attelle parce que le texte "reste initialement fermé à nos entreprises habituelles de compréhension» (p.31). Il s'agit dès lors de se forger un projet de lecture par le biais d'une métaphore fondatrice, de trouver une façon de lire le texte qui permette de faire signifier ses zones d'ombre.

Explorant sous divers aspects le couple classique compréhension-interprétation, Gervais montre que «le seuil de l'interprétation, ce n'est pas autre chose que celui de la lecture littéraire» (p.47).

L'interprétation est une «mise en relation (une correspondance), survenant en contexte, déclenchée par un prétexte, dont rien en soi ne garantit le respect ou encore la validité» (p.62). Tant que rien n'entrave la progression à travers le texte, on parle de compréhension. Mais lorsqu'on bute sur un aspect textuel dont la signification ne se laisse pas immédiatement saisir et qui exige un travail plus approfondi, on passe en interprétation:

[...] il y a interprétation [...] quand l'écart entre ce qui est attendu et ce qui survient est trop grand et qu'il se remarque explicitement comme différence, signe d'une étrangeté, manifestation d'une altérité. (p.59)

C'est là le prétexte de l'interprétation. La multiplicité des interprétations, sinon leur conflit, indique bien que l'acte d'interpréter prend appui sur des cadres de référence, des «savoirs et [des] systèmes de description» (p.67), des «façons d'organiser l'expérience" (p.68) divers et qui ne sont pas tous en accord. Ces cadres de référence sont les présupposés de l'acte d'interpréter, lui confèrent une stabilité et un sens et le régulent. Ce sont les contextes de l'interprétation. De même, la lecture littéraire

[...] est une relation réglée [...]; elle répond par conséquent à des règles qui déterminent souvent quels sont les prétextes à un investissement accru de la part du lecteur, et qui établissent ce qui vaut la peine d'être lu et commenté [...]. (p.72)

Activité d'exploration, d'éclaircissements sémiotiques, la lecture littéraire est donc une pratique qui remet en question les textes et les savoirs sur le texte en travaillant sur les zones d'ombre et selon des modalités où s'affirment la singularité et du lecteur, qui doit partir des difficultés qu'il rencontre et chercher à les faire signifier, et du texte lu, qui vaut pour lui-même, et non comme une actualisation parmi d'autres de réflexions théoriques ou méthodologiques générales.

\section{POUR PENSER L'AIRE DU DIRE:}

PARADIGMES ET JEUX DE LANGAGE

Conformément à nos hypothèses, nous voudrions maintenant proposer une manière de voir la lecture littéraire comme ouverture et délimitation de l'aire du dire. En d'autres termes, lorsqu'on se décide à faire une lecture littéraire, on se situe de fait dans une pratique sémiotique réglée qui permet et contraint ce qu'on va penser du texte sur lequel on se prépare à travailler. De même que les coups des joueurs d'échecs sont directement fonction des règles du jeu - et n'ont de pertinence que d'en être fonction-, de même la production d'une lecture littéraire a pour condition de possibilité et d'existence la façon dont la pratique est réglée.

C'est à mettre au point cette conception des pratiques sémiotiques que nous allons nous employer dans les lignes qui suivent, au gré d'un parcours sélectif chez deux auteurs. Nous trouverons dans les jeux de langage de Wittgenstein et les paradigmes de Kuhn une importante source d'inspiration pour envisager la lecture littéraire comme ouverture et délimitation de l'aire du dire. Au terme de cette excursion, nous devrions être en mesure d'asseoir solidement notre hypothèse initiale.

Dans La Structure des révolutions scientifiques, Kuhn utilise le terme "paradigme» dans deux acceptions différentes mais étroitement liées. Premièrement, le paradigme est tout ce qui fait que, à un moment donné, les scientifiques pratiquent telle discipline de telle façon; c'est ce

[...] que partagent [les] membres [d'une communauté scientifique et] qui explique la relative plénitude des communications sur le plan professionnel et la relative unanimité des jugements sur le plan professionnel.

(1983: 248) 
Deuxièmement, les paradigmes sont ces exemples sur la base desquels les étudiants se familiarisent avec une théorie donnée. Le travail sur les exemples permet aux étudiants d'acquérir cet ensemble organisé de concepts et cette manière de voir le monde qui sont partie intégrante du paradigme dans sa première acception. Ce sont ces deux aspects de la notion de paradigme et leurs relations que nous nous proposons maintenant de détailler. On aura sans doute compris que, dans notre esprit, des liens très étroits se tissent entre d'une part paradigme, travail scientifique et monde chez Kuhn et, d'autre part, lecture littéraire comme pratique sémiotique.

La première acception du terme "paradigme" ne se laisse pas aisément saisir dans le texte kuhnien: elle ne fait pas l'objet d'une définition qui la découperait avec précision et ce qu'elle recoupe semble de dimensions variables au gré de ses apparitions dans l'argumentation. Aussi allons-nous essayer, par des citations du texte de Kuhn, de donner une idée de ce qu'est le paradigme au lieu d'en fournir une définition claire.

Voici pour commencer trois fragments où il est question de changement de paradigme - de révolution scientifique. Indiquant ce qui se modifie lors d'un tel changement, Kuhn montre certains traits importants du concept. Dans le premier extrait, Kuhn cite un historien qu'il qualifie lui-même de «clairvoyant»:

On manipule les mêmes faits qu'auparavant mais en les plaçant l'un par rapport à l'autre dans un système de relations qui est nouveau parce qu'on leur a donné un cadre différent. (p. 124)

Plus loin, il précise cette idée de cadre:

[...] l'apparition d'une nouvelle théorie brise une tradition de recherche scientifique et en introduit une nowvelle, conduite selon des règles différentes, dans le cadre d'un univers discursif différent [...]. (p.125)

D'un point de vue général, enfin, on peut se figurer le passage d'un paradigme à un autre comme «un déplacement du réseau conceptuel à travers lequel les hommes de science voient le monde» (p. 147). On voit ici à l'œuvre la force organisatrice du paradigme: il organise le regard porté sur les faits, les faits euxmêmes et enfin la façon dont le scientifique traite ces faits. En une première approximation, on pourrait dire que le monde dans lequel et sur lequel travaille un homme de science est ce qu'il est dans la mesure où il est appréhendé par le biais du paradigme. Mais il faut aller plus loin, si l'on veut respecter l'esprit du texte de Kuhn: un paradigme n'est pas, par exemple, ce qui permet à un scientifique de voir des atomes. Il n'est pas entre l'homme de science et le monde; ce sont plutôt, si l'on veut, l'homme de science et le monde qui sont en lui. Cette formulation apparemment ésotérique signifie d'une part que l'homme de science est tel, pense ce qu'il pense, agit comme il le fait parce qu'il a parfaitement intégré toute la manière de penser, de voir, de dire et de faire impliquée par un paradigme donné. Elle signifie d'autre part que le monde sur lequel travaillent les scientifiques n'est pas une base stable interprétée dans un second temps de multiples manières: le monde des physiciens quantiques, par exemple, est ce qu'il est dans la mesure où l'organisation conceptuelle du paradigme l'a formaté, l'a découpé, y a isolé des éléments, les a nommés conformément à une vue d'ensemble, les a reliés entre eux, etc. ${ }^{3}$. Le simple fait d'identifier un atome (étymologiquement, on le sait, in-divisible, premier) implique avec lui l'entier d'un paradigme, toute une architecture conceptuelle.

Le point mérite d'être souligné: au cœur du paradigme se trouve un ensemble organisé de concepts plus ou moins définis. Pour des raisons qui deviendront claires lorsqu'on abordera la seconde acception du paradigme, les concepts ne sont pas tous nécessairement définis, et, s'ils le sont, rien ne prouve qu'ils soient précis. Il ne s'agit pas non plus d'un concept, mais d'un ensemble organisé de concepts: c'est-à-dire plusieurs concepts interdéfinis, selon des liens dont les modalités restent à déterminer dans chaque cas. C'est la spécificité de l'organisation conceptuelle qui ouvre et délimite l'aire du faire scientifique: les hypothèses et travaux scientifiques sont considérés comme pertinents, dans la mesure où 
ils s'inscrivent sur l'arrière-fond de possibilités déterminé par l'agencement des concepts.

Il faut également insister sur l'organisation de la perception par le paradigme. La citation suivante nous permettra d'aborder cet aspect de la problématique:

[...] les adeptes de paradigmes concurrents se livrent à leurs activités dans des mondes différents. [...] Travaillant dans des mondes différents, les deux groupes de scientifiques voient des choses différentes quand ils regardent dans la même direction à partir du même point. [...] dans certains domaines ils voient des choses différentes, et ils les voient dans un rapport différent les unes par rapport aux autres. (p.207)

Lorsqu'il identifie un objet du monde sur lequel il veut travailler, l'homme de science ne se livre pas à une activité préalable à la recherche au sein d'un paradigme: c'est déjà le paradigme qui lui permet d'identifier l'objet en question, qui le lui montre, relativement à son organisation conceptuelle, sous un aspect pertinent et qui le met dans un rapport particulier avec d'autres objets. Lorsque Kuhn parle de «mondes différents», il faut bien voir qu'il s'agit non pas de l'entier de l'expérience d'un être humain, mais bien de la portion de réel sur quoi porte le travail du scientifique. Et cette portion de réel peut être organisée de façons diverses par divers paradigmes, et les objets qui la composent apparaittre sous divers aspects et être reliés de diverses manières.

Le paradigme fait plus que donner une forme aux objets du travail scientifique, voire les faire apparaître:

[...] les paradigmes fournissent aux scientifiques non seulement une carte, mais aussi certaines directives essentielles à la réalisation d'une carte. En apprenant un paradigme, l'homme de science acquiert à la fois une théorie, des méthodes et des critères de jugement, généralement en un mélange inextricable. C'est pourquoi, lors des changements de paradigme, il y a généralement déplacement significatif des critères déterminant la légitimité des problèmes et aussi l'acceptabilité des solutions. (p.155)

On peut partiellement reformuler ainsi ce fragment: le paradigme organise le monde du scientifique et ce que ce dernier peut en dire et y faire. De ceci, la détermination de la légitimité des problèmes et de l'acceptabilité des solutions nous paraît exemplaire. L'architecture conceptuelle d'un paradigme et la structure qu'il confère de fait au monde ouvrent en le limitant un éventail de questions qui peuvent être posées à l'objet d'étude et d'actions qui peuvent être entreprises: en physique quantique, il y a lieu de s'interroger sur la vitesse ou la position de telle particule; essayer de déterminer sa couleur, par contre, n'est pas une question pertinente, pas plus que de savoir si elle émet des bruits. À l'autre bout de la chaîne et pour les mêmes raisons, le paradigme circonscrit les possibilités de réponses: visant la pertinence, les solutions doivent déployer un argument qui s'inscrit dans l'aire du paradigme - faute de quoi elles paraîtront excentriques.

On aura saisi l'étendue de ce qui relève du paradigme dans sa première acception, ainsi que sa force contraignante. Pour ne pas donner de cette dernière une fausse image, nous voudrions toutefois glisser quelques mots avant de passer à la seconde acception du paradigme. Nous aurions en effet failli à notre tâche si l'on venait maintenant à se représenter le paradigme comme une voix impérieuse et les scientifiques comme de simples scribes dociles. Tout différemment, le paradigme, par son architecture conceptuelle, ouvre et rend possible un espace d'objets, de discours et d'actions au sein duquel les hommes de science conduisent une infinité d'observations, d'expériences et produisent une infinité de discours mais pas n'importe lesquels. La contrainte du paradigme laisse ainsi une marge de manœuvre considérable. Plus encore: il faut se rendre compte que l'absence d'une organisation conceptuelle contraignante aurait pour effet non pas un élargissement significatif du domaine des possibles actionnels et discursifs, mais sa disparition pure et simple - puisque ce domaine doit son existence au paradigme.

La seconde acception du paradigme considère le problème sous l'angle, différent mais complémentaire, de l'intégration d'un réseau conceptuel - le paradigme étant maintenant les exemples et problèmes sur lesquels les étudiants travaillent pour devenir hommes de science. Dans sa première acception, on s'en souvient, le 
paradigme est de l'ordre de l'implicite; comment dans ces conditions peut-il être appris? C'est à cette question que la seconde acception du paradigme veut répondre.

Dans la mesure où l'organisation conceptuelle qu'est le paradigme dans sa première acception ne se laisse pas réduire à un argument, à une thèse ou à un ensemble de règles explicites, il ne s'apprend et ne se comprend pas comme on peut apprendre et comprendre une argumentation ou un corpus de règles. Il s'apprend plutôt par le biais d'un travail répété sur des problèmes et sur des expériences en laboratoire. Kuhn ne s'attarde pas à ce processus assez complexe à envisager et nous ne substituerons pas notre pensée à la sienne. Notons néanmoins qu'un problème, par exemple, de physique quantique est dès l'abord formulé en symboles, qui prennent sens en raison de leur appartenance au réseau conceptuel de la physique quantique, et ne peut être résolu que par le biais d'un raisonnement situé sur cet arrière-fond: essayer de résoudre ce genre de problème implique de se confronter directement et de manière soutenue au paradigme quantique. Il en va de même pour les expériences en laboratoire. De la sorte, on peut, nous semble-t-il, parler d'apprentissage par progressive familiarisation - mais une familiarisation qui passe par la bande dans la mesure où l'essentiel reste implicite. Que l'apprentissage soit ainsi dérivé a pour conséquence le caractère "tacite» de la connaissance du paradigme chez les hommes de science: en un sens, il informe leurs actions et leurs discours sans qu'ils en soient forcément conscients; il est ce qui va de soi, ce qui reste en marge de la pratique, à la périphérie de la recherche.

Retenons de ce bref détour par la notion de paradigme chez Kuhn qu'il est un réseau conceptuel implicite et flou qui ouvre en la délimitant l'aire du dire et du faire scientifiques, des problèmes légitimes et des solutions acceptables; qu'il donne un sens à la pratique et à ses instruments; qu'il est, à tous les sens du terme, une manière de voir une portion plus ou moins étendue de la nature; qu'il s'apprend par progressive familiarisation moyennant un travail sur des problèmes et des expériences en laboratoire.
Nous l'avons dit: les paradigmes chez Kuhn sont des concepts très proches des concepts de jeux de langage dans la seconde philosophie de Wittgenstein, et notamment dans De la certitude. L'ensemble de cette philosophie a pour objet de mettre en lumière les manières de voir qui fondent nos pratiques humaines, de la plus humble à la plus complexe.

Systématiquement, l'investigation prend appui sur des exemples de considérations psychologiques, mathématiques, historiennes, esthétiques ou autres et cherche à sortir de l'ombre les fondements conceptuels qui sont les conditions de possibilité de ces exemples concrets. Pour faire bref, on peut dire que les pratiques sont ce que Wittgenstein nomme «jeu de langage» - dans son acception tardive et élargie -, les exemples et expressions concrets sont les «coups» faits à l'intérieur d'un jeu de langage et l'arrière-fond conceptuel est la "grammaire» du jeu de langage. Nous pouvons ainsi dire que la lecture littéraire est un jeu de langage, qu'une lecture littéraire concrète, telle qu'on peut la voir dans une revue scientifique ou la produire, est une série de coups dans le jeu de langage de la lecture littéraire et que les concepts implicites de texte et de signification qu'elle véhicule et qui la permettent appartiennent à la grammaire du jeu de langage.

De la certitude est un texte complexe, entrelaçant plusieurs lignes argumentatives à géométrie variable; aussi, pour ne pas nous perdre, concentrerons-nous notre attention sur une seule des questions abordées par Wittgenstein. Le philosophe pose qu'on ne peut pas douter de tout et que, lorsqu'on joue un jeu de langage, on met par le fait même un certain nombre d'éléments à l'abri du doute. Ces éléments relèvent de la grammaire du jeu de langage, ou encore des moyens de la représentation. Ainsi, d'un élève qui, dans un cours d'histoire, interromprait constamment son professeur pour douter que la Terre ait existé il y a plus de cent ans, Wittgenstein dit:

[...] lui non plus n'a pas encore appris à poser des questions. Il n'a

pas appris le jeu que nous voulons lui enseigner. [...] Un doute de ce genre ne fait pas partie de ceux que nous connaissons dans ce jeu. (Mais ce n'est pas que nous ayons le choix de ce jeu). (1965: 85) 
Autrement dit, l'histoire comme jeu de langage implique une certaine manière de voir le monde, le temps, la causalité, etc. Cette manière de voir, cette architecture conceptuelle est la condition sine qua non pour que ce jeu puisse être joué: elle est ce qui permet le discours des historiens.

À l'image du paradigme kuhnien, cette manière de voir est silencieuse:

Il peut se faire par exemple que l'ensemble de notre recherche soit ainsi disposé que, de ce chef, certaines propositions, si jamais elles sont formulées, sont hors de doute. Elles gîtent à l'écart de la route sur laquelle se meut la recherche. (p.47)

Les propositions qui sont pour moi solidement fixées, je ne les apprends pas explicitement. Je peux les trouver après coup, comme je trouve l'axe de rotation d'un corps en révolution. L'axe n'est pas fixé au sens où il serait maintenu fixe, mais c'est le mouvement tout à l'entour qui le détermine comme immobile. (p.60)

Cette discrétion s'inscrit dans le cadre de la distinction entre dire et montrer, qui traverse toute la philosophie de Wittgenstein: lorsque, par exemple, on produit une lecture littéraire, on dit un certain nombre de choses sur un texte donné, et on en montre d'autres, comme une manière de considérer le texte littéraire et la signification en général. En d'autres termes, ce que montrent les coups d'un jeu de langage, c'est la grammaire du jeu de langage qui les rend possibles.

Abordant la fonction de ces architectures conceptuelles qui se montrent sans se dire, Wittgenstein propose l'exemple suivant, partiellement daté:

Tout ce que j'ai vu ou entendu contribue à me convaincre qu'il n'y a pas un homme qui se soit jamais éloigné de la terre. Rien dans mon image du monde ne parle pour une vue contraire.

Mais cette image du monde, je ne l'ai pas parce que je me suis convaincu de sa rectitude; ni non plus parce que je suis convaincu de sa rectitude. Non, elle est l'arrière-plan sur le fond duquel je distingue entre vrai et faux. Les propositions qui décrivent cette image du monde pourraient appartenir à une sorte de mythologie. Et leur rôle est semblable à celui des règles d'un jeu; et ce jeu, on peut aussi l'apprendre de façon purement pratique, sans règles explicites. (p.49)
Quel est le rôle des règles d'un jeu? On peut dire qu'elles interdéfinissent les éléments du jeu, qu'elles découpent un espace des possibles pour les coups à venir, qu'elles sont également ce en fonction de quoi les coups seront joués, ce qui leur conférera sens et légitimité. Ainsi en va-t-il des fonctions de l'image du monde citée dans l'exemple: cet arrière-plan permet de dire ce qui est dit, il détermine l'aire du dire. En retour, ce qui est dit ne peut l'être que du fait qu'il est situé sur cet horizon qu'il montre par chacune de ses occurrences ${ }^{4}$.

La complexité du rapport qui se laisse entrevoir entre un coup dans un jeu de langage et tout l'arrièrefond qui le permet est également soulignée à plusieurs reprises par Wittgenstein:

Toute vérification de ce qu'on admet comme vrai, toute confirmation ou infirmation prennent déjà place à l'intérieur d'un système. Et assurément, ce système n'est pas un point de départ plus ou moins arbitraire ou douteux pour tous nos arguments; au contraire, il appartient à l'essence de ce que nous appelons un argument. Le système n'est pas tant le point de départ des arguments que leur milieu vital. (p.51)

«Si nous commençons à croire quelque chose, ce n'est pas une proposition isolée mais un système entier de propositions» (p. 57-58). Le système dont il est ici question recoupe les articulations conceptuelles qui composent la grammaire d'un jeu de langage. S'il y a un argument, dit le philosophe, alors il y a derrière lui tout un système qui est véritablement la condition d'existence des arguments particuliers. Il n'y a somme toute rien d'isolé: un argument est toujours un coup dans un jeu de langage et prend sens à l'intérieur de celui-ci. Le terme «argument» ne doit toutefois pas laisser penser que ce qui est dit ici ne vaut que pour une argumentation. Est en jeu tout ce qui peut se passer dans n'importe quel jeu de langage: lorsque, jouant un jeu de langage particulier, un individu fait un coup - quelle que soit sa forme - ce coup est rendu possible par - et donc implique - l'entier de la grammaire du jeu de langage en question.

En s'éclairant réciproquement et en approfondissant chacun des points complémentaires, 
Kuhn et Wittgenstein esquissent une manière de penser les pratiques humaines très heuristique, et au sein de laquelle il nous semble y avoir un gain substantiel à envisager les pratiques visant à élaborer de la signification. L'un et l'autre estiment que ce qui se fait et se dit doit son existence, son sens et sa légitimité à son inscription au sein d'une (ou de plusieurs) pratique(s) réglée(s); pratique réglée dans la mesure où, d'une part, elle régule le faire et le dire et où, d'autre part, elle est constituée d'un ensemble de concepts implicites interdéfinis. Cette architecture conceptuelle est intégrée par les usagers d'une pratique et informe leur manière de voir, de dire et de penser tel aspect de leur expérience.

\section{SINGULARITÉ DE LA PRATIQUE}

VERSUS SINGULARITÉ DU LECTEUR

À la lumière de la manière de considérer les pratiques sémiotiques que Kuhn et Wittgenstein nous ont permis d'esquisser, la question de la singularité et de la responsabilité du lecteur littéraire peut être reposée et recevoir un éclairage nouveau. Rappelons cette évidence que la singularité en question ne saurait être absolue: ce n'est pas n'importe quelle hypothèse de lecture qui sera acceptée comme valable, sous prétexte d'une mise à distance des impératifs théoriques et méthodologiques. Indépendamment du fait que certaines hypothèses seront jugées simplement plus élégantes ou plus heuristiques que d'autres (qui dans le fond sont tout aussi valables), certaines hypothèses seront considérées comme inacceptables. Singularité ne rime pas avec équivalence de toutes les hypothèses de lecture. Seulement, dire cela, c'est somme toute faire écho aux propos de Kuhn, lorsqu'il dit qu'un paradigme détermine l'acceptabilité des problèmes et des solutions - à ceci près qu'on escamote la présence du paradigme, de l'architecture conceptuelle qui ouvre l'espace du dire et du faire. En d'autres termes, refuser certaines hypothèses implique une pratique sémiotique conceptuellement organisée, de manière implicite et floue. Dire à quelqu'un qu'il propose une hypothèse farfelue, cela revient d'ailleurs souvent, sinon toujours, à ne pas être d'accord avec la manière de voir le texte ou l'élaboration de la signification que montre son hypothèse - de là, aussi, la difficulté qu'on éprouve à justifier clairement ce refus: on sent bien que sa manière de voir les choses et la nôtre divergent, mais, en raison de leur nature implicite et floue, on peine à les exprimer. Peut-être, d'ailleurs, ces hypothèses refusées devraient-elles faire l'objet d'une réelle réflexion théorique: si elles remettent effectivement en question les soubassements implicites constitutifs de la lecture littéraire comme pratique sémiotique, elles peuvent, dans le même geste et pour peu qu'on y accorde une attention soutenue, nous permettre de faire partiellement passer ces soubassements dans l'ordre de l'explicite. De plus, il n'est pas d'emblée exclu, même si la chose paraît pour le moins ardue, qu'elles nous amènent à réviser nos manières de faire sens, ou du moins qu'elles nous laissent entrevoir d'autres manières de faire sens. De ce point de vue, mais par un autre biais, les hypertextes fictionnels, en raison de leur inadéquation manifeste avec notre concept implicite de texte et nos manières ordinaires de faire sens, posent un véritable défi à qui veut les analyser. Et, de fait, on ne sait pas encore très bien quoi faire avec ces étranges objets récemment apparus dans la sphère littéraire ${ }^{5}$.

Dans ces conditions, qu'en est-il de la singularité et de la responsabilité du lecteur littéraire dans ses choix épistémologiques? Nous pensons que la part de singularité et de responsabilité est limitée. Elle est limitée par le seul fait de vouloir faire une lecture littéraire: la lecture littéraire est une pratique sémiotique, une manière spécifique de faire sens, qui ouvre en la délimitant l'aire du dire que le lecteur pourra ensuite parcourir à sa guise. La singularité et la responsabilité du lecteur sont celles que lui laisse la pratique - et c'est ainsi et seulement ainsi qu'elles peuvent exister et posséder une certaine valeur. L'absence d'impératifs théoriques ou méthodologiques stricts n'hypothèque en rien la présence des éléments implicites contraignants constitutifs de la pratique, condition et légitimation des significations élaborées et des hypothèses proposées. Concédera-t-on que la marge de manouvre du lecteur est plus grande que 
dans une lecture savante? On le pourrait, mais au prix fort d'une réduction de la spécificité de la lecture littéraire; nous aimerions mieux dire que la marge de manœuvre est autre, parce que la pratique est autre et que, principalement, les modalités d'élaboration de la signification et les opérations envisageables sur le texte sont d'une autre nature.

Par ailleurs, est-on davantage responsable de ses choix épistémologiques en lecture littéraire? Pour que ce soit fondamentalement le cas, il faudrait être pleinement conscient de l'entier de l'architecture conceptuelle implicite de la pratique et se déclarer en accord avec elle. À la suite de notre périple à travers Kuhn et Wittgenstein, on doutera de la possibilité d'une telle réflexion épistémologique. Comme on l'a vu, c'est à la suite d'un apprentissage, et par la bande, que cette architecture s'acquiert. De plus, une fois qu'elle est acquise, elle informe durablement et sans qu'il y ait de décision consciente le dire et le faire. Pour forcer la note, nous irons jusqu'à affirmer que, pour faire une lecture littéraire, il n'est pas particulièrement utile d'être conscient des manières implicites de voir le texte et de l'élaboration de la signification véhiculées par la pratique.

Par contre, le propre d'une réflexion sur la réception nous semble être justement d'apporter des éclaircissements sur les manières de voir qui ont délimité l'aire dans laquelle la lecture s'est inscrite. De là, et dans un cadre sémiotique, s'ouvre tout un jeu de perspectives et de possibilités pour cette investigation. Compte tenu de l'importance accordée par Kuhn et Wittgenstein à l'apprentissage, et compte tenu de leur insistance à inscrire respectivement le jeu de langage dans une forme de vie et le paradigme dans un environnement culturel, il y a moyen de mener nombre d'enquêtes dans des directions variées: la mise en évidence des manières de voir liées à certaines écoles critiques, les modalités de transmission de ce savoir spécifique, l'intégration d'une manière de voir le texte dans un espace socioculturel et les interactions entre ces deux aspects, les modifications effectivement apportées dans les manières de voir le texte par des approches qui se veulent novatrices, etc. ${ }^{6}$.

\section{CONCLUSION}

Dans l'introduction du présent article, nous parlions de la singularité du lecteur littéraire en termes de singularité réglée. Peut-être comprend-on maintenant mieux pourquoi. Nous pensons préserver l'originalité de cette pratique et la situer au plus près de certains enjeux essentiels du travail sur le texte. Situer cette singularité au sein d'un espace ouvert et délimité par l'architecture conceptuelle véhiculée par la lecture littéraire revient à la faire dériver des modalités d'établissement de la signification propres à cette pratique sémiotique. Dans le même temps, cela revient à mettre l'accent sur la singularité de la pratique, ses paramètres sémiotiques idiosyncratiques - car c'est là que réside pour nous tout son intérêt. L'accent mis sur la singularité et la responsabilité du lecteur découle sans doute de la volonté de positionner la lecture littéraire en regard d'autres pratiques fondées sur des méthodologies fortes ou des impératifs théoriques particuliers: cette géographie intellectuelle met peut-être en lumière certains traits de la lecture littéraire - mais ceux seuls qui ont une commune mesure avec les autres pratiques évoquées, et non nécessairement ceux qui sont au cour de la lecture littéraire.

Plus fondamentalement encore, la lecture littéraire nous apprend quelque chose d'essentiel sur notre rapport aux textes: on voit en effet que le travail sur les textes, même en dehors de partis pris théoriques ou méthodologiques, n'est possible et n'a de sens qu'en tant qu'il se situe au sein d'une aire ouverte par une pratique spécifique qui véhicule minimalement une manière de voir le texte, les opérations envisageables sur le texte et les modalités d'élaboration de la signification, c'est-à-dire une manière de voir l'objet sur lequel on travaille, le type de travail qu'on peut effectuer sur lui et les types de questions qu'on peut poser et de réponses qu'on peut donner ${ }^{7}$. On est ainsi à même de mesurer la spécialisation, la complexité conceptuelle et la richesse de cet acte à la familiarité trop souvent trompeuse. Du coup, on est à même de percevoir la spécificité de cette sémiotique de la réception sur l'horizon de laquelle prennent sens 
nos propos. Par-delà le clin d'œil parodique, nous voulions essayer de montrer le gain à retirer d'un croisement entre la problématique de la réception et une conception sémiotique proposant un mode particulier d'investigation des pratiques humaines destinées à faire sens, où cette action est perçue comme réglée par une architecture conceptuelle floue, apprise dans un cadre éducatif particulier et intégrée à un ensemble socioculturel où elle entre en relation avec un ensemble d'autres activités, dans des domaines divers. Il appartient maintenant à la réception de notre entreprise de juger de son opportunité.

\section{N O TES}

1. Le présent article poursuit des réflexions personnelles commencées dans un séminaire animé à l'Université du Québec à Montréal par B. Gervais et M. Roy et intitulé "Lecture typique et lecture atypique", durant une année où j'ai bénéficié de subsides de la Société Académique Vaudoise et de la Fondation de l'Université du Québec à Montréal. 2. Pour être marginale dans le champ théorique de la réception, cette entreprise n'est pas nouvelle non plus. On peut par exemple la rapprocher de celle de Wlodzimierz Bolecki (1983). Dans une veine kantienne et historienne, il envisage ainsi la question : «Il s'agit d'un problème concernant les "possibilités" de lecture qui englobe les conditions a priori de la lecture des œuvres littéraires dans une période donnée [...]» (p. 77); il vise à mettre en lumière «l'ensemble de l'appareil esthético-cognitif grâce auquel le texte peut être examiné». Pour lui, «la science de la littérature commence là où elle nous révèle le langage que nous utilisons pour parler d'elle tout en nous indiquant les cadres qui limitent ce langage» (p. 81). Les parallèles sont nombreux entre sa démarche et celle que nous allons entreprendre, et sa perspective diachronique complète bien notre visée pour l'instant synchronique. Toutefois, notre cadre théorique nous amène à borner notre réflexion au domaine conceptuel et à nous défier des ensembles normatifs trop stricts. 3. Profitons de cet exemple pour insister sur le fait que, ni dans la pensée de Kuhn ni dans la nôtre, il n'existe un seul et unique paradigme scientifique. Pour aller plus loin, affirmons qu'il n'existe pas, par exemple, un paradigme en physique: il faut plutôt dire que la physique newtonienne est un paradigme, que la physique einsteinienne en est un autre et que la physique quantique en est un troisième. La pensée de Kuhn relativement au paradigme se développe dans deux directions : la première, synchronique, cherche à définir, avec les nuances qu'on a apportées, le paradigme; la seconde, diachronique, cherche à montrer comment un paradigme s'impose, guide la recherche, puis est éventuellement contesté et enfin remplacé par un autre paradigme. Les dimensions de la présente réflexion et des ambitions de cohérence argumentative qu'on jugera avec bienveillance nous poussent à privilégier la première voie; en fin de parcours, toutefois, nous proposerons quelques ouvertures en direction de la seconde.

4. On aura noté en passant le contexte d'apprentissage mentionné dans cette citation et dans la citation précédente, où se dessine, ici comme ailleurs, une nette parenté entre Kuhn et Wittgenstein. Il y aurait d'ailleurs lieu de se demander si le contexte d'apprentissage n'est pas particulièrement propice aux réflexions sur les pratiques sémiotiques telles que nous les envisageons à la suite des deux penseurs, dans la mesure où c'est sans doute le seul moment où peut être concrètement questionnée l'aire du dire et où peut avoir lieu une défamiliarisation permettant la mise en évidence de certains aspects fondamentaux d'une pratique sémiotique.

5. Voir à ce propos B. Gervais et N. Xanthos (à paraître). Par les deux bouts de la chaîne, les hypothèses de lecture refusées et les hypertextes fictionnels remettent en question nos pratiques sémiotiques conventionnelles: d'un côté en s'attaquant à leurs concepts implicites fondateurs, de l'autre en opposant auxdits concepts un monde qu'ils ne sont pas en mesure de rendre signifiant. Ce deuxième cas de figure apporte d'ailleurs un judicieux contrepoids à notre argument qui aurait pu jusque-là laisser croire que le monde se laisse passivement informer par nos pratiques sémiotiques; on entrevoit maintenant des possibilités d'interaction bienvenues.

6. Sur ce point aussi, nous renvoyons notre lecteur à Bolecki qui explore cette dimension socio-historique, seulement effleurée ici: «La mise au clair du fonctionnement d'un style de réception doit renvoyer aux cadres sociaux de lecture traités comme des configurations des normes propres à une époque d'une part, et au type de lecture constituant un corps de principes plus fondamentaux que ceux qui se manifestent dans la lecture d'une œuvre isolée, de l'autre» (1983: 80). Pour la réflexion sur les transformations de la manière de voir le texte causées par l'apparition de nouvelles théories, la révolution scientifique telle que la présente Kuhn est naturellement une base de premier ordre.

7. La réflexion que mène M. Charles (1995) mérite à ce titre d'être citée. Dans une optique légèrement différente de la nôtre, il essaie de voir quels sont les présupposés que l'on possède sur le texte, et la façon dont ces présupposés informent le travail critique, la méthode, la théorie et les outils mis au point. Aux présupposés traditionnels de cohérence et d'unité du texte, il cherche à opposer une autre vision de l'objet, fondée non plus sur la recherche du même à l'intérieur du texte, mais sur celle de l'autre, sur l'observation des lieux où la cohérence est mise en péril plutôt que sur celle des lieux où elle se manifeste - étant entendu que la mise en péril et la manifestation de la cohérence sont toutes deux construites par l'analyste et non données dans le texte. L'avenir dira si la théorie et la méthodologie élaborées trouveront un large écho, mais on peut d'ores et déjà s'inspirer des conclusions épistémologiques de l'auteur.

\section{RÉFÉREN CES BIBLIO G RAPH IQ U ES}

BOLECKI, W. [1983]: «L'Espace socioculturel et la lecture», dans J. Heisten (dir.), La Réception de l'œuure littéraire (Actes du Colloque de l'Université de Wroclaw), Pologne, Romanica Wratislaviensia. ChARLES, M. [1995]: Introduction à l'étude des textes, Paris, Seuil. GERVAIS, B. [1997]: Lecture littéraire et explorations en littérature américaine, Montréal, XYZ.

GERVAIS, B. et N. XANTHOS [à paraître] : «L'Hypertexte: une lecture sans fin ", Littérature, informatique, lecture, dirigé par M. Lenoble et A. Vuillemin, Limoges, PULIM.

KuHn, T. [1983]: La Structure des révolutions scientifiques, Paris,

Flammarion.

SCHOBER, R. [1983]: «Esthétique de la réception et lecture réaliste», dans J. Heisten (dir.), La Réception de l'œeuvre littéraire (Actes du Colloque de l'Université de Wroclaw), Pologne, Romanica Wratislaviensia. WITTGENSTEIN, L. [1961]: Tractatus logico-philosophicus, suivi de Investigations philosophiques, Paris, Gallimard;

[1965]: De la certitude, Paris, Gallimard. 